Bull. Korean Math. Soc. 51 (2014), No. 2, pp. 539-545

http://dx.doi.org/10.4134/BKMS.2014.51.2.539

\title{
LOG-CONCAVITY AND ZEROS OF THE ALEXANDER POLYNOMIAL
}

\author{
Alexander Stoimenow
}

\begin{abstract}
We show that roots of log-concave Alexander knot polynomials are dense in $\mathbb{C}$. This in particular implies that the log-concavity and Hoste's conjecture on the Alexander polynomial of alternating knots are (essentially) independent.
\end{abstract}

\section{Introduction}

In this paper we will treat the Alexander polynomial $\Delta(t)$ of knots. Below $\Delta \in \mathbb{Z}\left[t, t^{-1}\right]$ will be normalized so that

$$
\Delta(1 / t)=\Delta(t) \quad \text { and } \quad \Delta(1)=1 .
$$

It is very well known what Alexander polynomials occur for an arbitrary knot: the conditions (1) precisely characterize such polynomials. (We will call the first property reciprocity) The question about those polynomials occurring for an alternating knot is much harder, and relates to several conjectures which have been treated over a period of time.

Some properties have been known. Let max $\operatorname{deg} \Delta$ be the maximal degree of $\Delta$ (maximal power of $t$ with non-zero coefficient). Because of reciprocity the minimal degree of $\Delta$, defined analogously, is min $\operatorname{deg} \Delta=-\max \operatorname{deg} \Delta$. Crowell and Murasugi $[2,10]$ proved that when $K$ is alternating, the polynomial $\Delta_{K}(t)$ is alternating, i.e., all coefficients of $\Delta_{K}(-t)$ are positive or all are negative. We call such polynomials also positive resp. negative. This is to mean in particular that all coefficients between min $\operatorname{deg} \Delta$ and $\max \operatorname{deg} \Delta$ are non-zero. To avoid ambiguities, let us assume that all Alexander polynomials we treat from now on have this property (which is not automatic).

The work of Crowell-Murasugi shows also that for $K$ alternating, the maximal degree of $\Delta$ gives the genus $g(K)$ (and canonical genus $g_{c}(K)$ ) of $K$ :

$$
g_{c}(K)=g(K)=\max \operatorname{deg} \Delta_{K} .
$$

Received January 3, 2013.

2010 Mathematics Subject Classification. Primary 57M25; Secondary 11C08.

Key words and phrases. genus, alternating knot, Alexander polynomial, dense, polynomial root. 
We are here in particular motivated by two conjectures. One is the logconcavity conjecture made in [14]. Let $[X]_{k}$ for $k \in \mathbb{Z}$ be the coefficient of $t^{k}$ in a Laurent polynomial $X \in \mathbb{Z}\left[t^{ \pm 1}\right]$. Call a polynomial $X$ to be log-concave, if $[X]_{k}$ are $\log$-concave, i.e.,

$$
[X]_{k}^{2} \geq[X]_{k+1}[X]_{k-1} \geq 0
$$

for all $k \in \mathbb{Z}$. (We assume the non-negativity of these expressions for technical reasons: we want to regard only positive and alternating polynomials as logconcave)

Conjecture 1 (log-concavity conjecture). If $K$ is an alternating knot, then $\Delta_{K}(t)$ is log-concave.

The log-concavity conjecture is a natural strengthening of a much older conjecture formulated by Fox, which is now referred to also as 'Trapezoidal' conjecture.

Conjecture 2 (Fox's Trapezoidal conjecture). If $K$ is an alternating knot, then there is a number $0 \leq n \leq g(K)$ such that for $\Delta_{[k]}:=\left|\left[\Delta_{K}\right]_{k}\right|$ we have

$$
\begin{array}{ll}
\Delta_{[k]}=\Delta_{[k-1]} & \text { for } 0<|k| \leq n, \\
\Delta_{[k]}<\Delta_{[k-1]} & \text { for } n<|k| \leq g(K) .
\end{array}
$$

We call polynomials of this form trapezoidal. Since log-concave polynomials are trapezoidal, the log-concavity conjecture implies Fox's conjecture.

The Trapezoidal conjecture has received some attention in the literature. It was verified for rational (2-bridge) knots [4] (see also [1]) and later for a larger class of alternating algebraic knots [11]. More recently, Ozsváth and Szabó used their knot Floer homology [12] to derive a family of linear inequalities on the coefficients of $\Delta$ for an alternating knot. For genus $g=2$ their inequalities are very similar to (and slightly stronger than) the Trapezoidal conjecture, and also settle (for general $g$ ) in (4) the case $|k|=g(K)$. In-Dae Jong [6, 7] has proved independently the Trapezoidal conjecture up to genus 2 using the generator description in [15], and observed that for genus 2 the log-concavity of $\Delta$ easily follows from trapezoidality. Then, in [13] the proof of log-concavity was extended to genus at most 4 using similar techniques.

The other conjecture motivating the present note is attributed to Hoste. I learned about it from personal communication with Murasugi.

Conjecture 3 (Hoste's conjecture). If $z \in \mathbb{C}$ is a root of the Alexander polynomial of an alternating knot, then $\Re e z>-1$.

The conjecture is true for special alternating knots. In this case all roots lie on $S^{1}$, the set of unit norm complex numbers (and -1 is not a zero of the Alexander polynomial of any knot). For a clarification see [3, 14]. In [13] we use again our generator classification and an appropriate calculation to prove Hoste's conjecture up to genus 4. 
The zeros of $\Delta$ seem less easy to control than the coefficients, and Hoste's conjecture is open even for general rational knots. This case was recently studied by L. Lyubich and Murasugi [8]. They obtained several results on the (real part of) the zeros of the Alexander polynomial of rational knots (and links), confirming Hoste's conjecture for some subclass of them.

Murasugi told that during his work Mizuma showed him the polynomial

$t^{-6}-2 t^{-5}+4 t^{-4}-8 t^{-3}+16 t^{-2}-32 t^{-1}+43-32 t+16 t^{2}-8 t^{3}+4 t^{4}-2 t^{5}+t^{6}$,

which has a zero $z$ such that $\Re e z<-1$. This shows that trapezoidality (or logconcavity) of an Alexander polynomial does not imply Hoste's conjecture. The above example is not too surprising, and the aim of this note is to generalize its intuition to a meaningful statement.

Theorem 1. The zeros of log-concave alternating Alexander knot polynomials are dense in $\mathbb{C}$.

This shows that statements regarding coefficients and such about zeros of Alexander polynomials are almost independent. There are minor relations, e.g., an alternating polynomial cannot have a real negative zero. There are also conditions arising when restricting the degree of the polynomial. For genus 2, Murasugi has verified that any alternating Alexander polynomial satisfies Hoste's conjecture.

Remark 1. Note that by the refinement of the realization result for Alexander polynomials in [16], we may approximate every complex number by zeros of log-concave alternating polynomials of knots with special properties, like hyperbolicity and (2) (both enjoyed by alternating knots, as known from [2, 10] resp. [9]), and arborescence.

Compare Theorem 1 also to the result in [5] regarding the Jones polynomial roots.

\section{Proof of Theorem 1}

The proof is short and elementary.

To avoid technical overhead, let us first change the variable $t$ to $-t$. Then we must approximate every complex number $z$ by zeros of reciprocal positive log-concave polynomials $Q$ with $Q(-1)=1$.

In this proof we will use $i=\sqrt{-1}$ exclusively for the imaginary unit. We fix a number $z \in \mathbb{C}$ which we would like to approximate. By density and reciprocity in (1), we may assume that $|z|>1$ and $z /|z|$ is not a root of unity, i.e., $\arg z \in[0,2 \pi)$, defined from $z=|z| e^{i \arg z}$, is not a rational multiple of $\pi$.

We will consider below polynomials (with real coefficients)

$$
Z_{l}(t)=|z|^{l}+|z|^{l-1} t+\cdots+|z| t^{l-1}+t^{l}=\frac{t^{l+1}-|z|^{l+1}}{t-|z|}
$$


We first realize $z$ as a zero of a polynomial $P_{k, l} \in \mathbb{R}[t]$,

$$
P_{k, l}=\alpha+\beta t^{k}+\gamma Z_{l}(t),
$$

where $\alpha, \beta, \gamma$ are positive real numbers, and $0<k<l$.

It will be important for us to observe that such $P_{k, l}$ can, for fixed proper $k$, be chosen with $l$ arbitrarily large, and so that $\max (\alpha, \beta) / \gamma$ grows slower than $|z|^{l}$, i.e.,

$$
\frac{\max (\alpha, \beta)}{\gamma} \leq \varepsilon \cdot|z|^{l},
$$

for every fixed $\varepsilon>0$. How to chose this $\varepsilon$ will become clear below.

To obtain $P_{k, l}$, first choose (and fix for the rest of the proof) $k$ so that

$$
\arg z^{k} \in\left(\arg \left(\frac{i}{z-|z|}\right)-\pi, \pi\right) .
$$

(Such a condition is indicated by the shaded region in the below figure.) Note that the condition is satisfiable, since $\frac{i}{z-|z|}$ has negative imaginary part (and thus the interval on the right of (7) is non-empty), and since $z /|z|$ is not a root of unity, i.e., $\left\{\arg z^{k}\right\}$ is dense in $[0,2 \pi)$.

Next choose, with the same justification, $l$ large enough so that $(z /|z|)^{l+1}$ is arbitrarily close to 1 and $\arg z^{l+1}<\pi$. Then

$$
\arg \left(z^{l+1}-|z|^{l+1}\right)=\arg \left(\left(\frac{z}{|z|}\right)^{l+1}-1\right) \rightarrow \frac{\pi}{2} .
$$

Thus with (7) we have that 0 lies in the triangle with vertices (i.e., convex hull of) $A=1, B=z^{k}$ and $C=Z_{l}(z)$ when $l$ is large enough. This gives $P_{k, l}$ (with $\alpha+\beta+\gamma=1)$ satisfying $P_{k, l}(z)=0$.

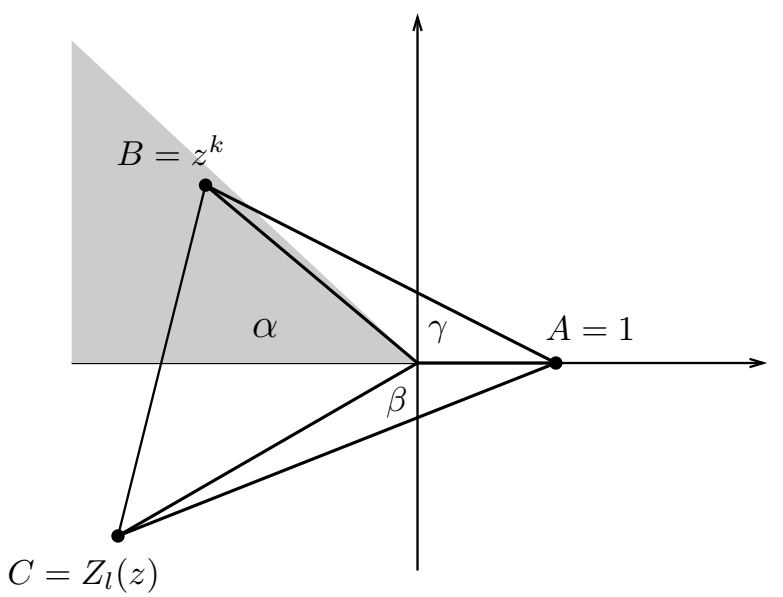

It remains to see (6). For this, for example, recall that $\alpha, \beta, \gamma$ have the ratio of the triangle areas shown in the figure with the same letter. The sides of 
$\triangle A B C$ have bounded distances to the origin, and the lengths of $A C$ and $B C$ grow slower than $|z|^{l}$, while this of $A B$ is fixed.

Lemma 1. Let $P_{k, l}$ be a polynomial like in (5) with (6). Consider

$$
P(t)=P_{k, l}(1 / t) \cdot P_{k, l}(t) .
$$

Then $P$ is strictly log-concave for l large enough.

(Strictly log-concave is to mean that if the first inequality in (3) becomes an equality, then both expressions vanish.)

We will prove the lemma in a moment, but let us first remark that after this lemma is proved, we are easily done.

Lemma 2. If $P$ is positive and (strictly) log-concave, so is $P(t) \cdot(1+t)$.

Proof. Let $a, b, c, d$ be four consecutive coefficients of $P$. Then we must show $(a+b)(c+d)<(b+c)^{2}$ from $a c<b^{2}$ and $b d<c^{2}$. Expanding the claim and simplifying using the two premises leads to $a d<b c$, which in turn follows from their product.

Let $\langle X\rangle$ mean the polynomial in $\mathbb{Z}\left[t^{ \pm 1}\right]$ obtained by rounding (say, everywhere up) all coefficients of $X \in \mathbb{R}\left[t^{ \pm 1}\right]$. Then choose $k, l$ large enough so that $P$ in (8) to be strictly log-concave. Fix these $k, l$ and consider

$$
Q_{q}=\langle q \cdot P\rangle \cdot t^{-1} \cdot(1+t)^{2}+1
$$

for $q \in \mathbb{N}$ with $q \rightarrow \infty$. Then $Q_{q}$ are integer reciprocal polynomials with $Q_{q}(-1)=1$, and so $Q_{q}(-t)$ are Alexander knot polynomials. Since $Q_{q} / q \rightarrow \tilde{P}$ converge coefficient-wise to the limit $\tilde{P}=P \cdot(1+t)^{2} / t$ (and $Q_{q}, \tilde{P}$ are of the same degree), strict log-concavity of $P$ and Lemma 2 imply (strict) logconcavity of $Q_{q}$ for $q$ large enough. Moreover, the same convergence implies (see Lemma 2 in [14]) that zeros of $Q_{q}$ approach the zero $z$ of $P$ (and of $\tilde{P}$ ).

The proof of Theorem 1 is thus concluded with the one of Lemma 1.

Proof of Lemma 1. Because of (6), and $k$ is chosen before $l$ and $\varepsilon$, the polynomials $P_{k, l}$ can be regarded as a perturbation of $Z_{l}$, in the sense that

$$
1 \leq \frac{\left[P_{k, l}\right]_{n}}{\left[Z_{l}\right]_{n}} \leq 1+\varepsilon
$$

for all $0 \leq n \leq l$. Here $\varepsilon$ is fixed before choosing $l$, and so is independent of $n$ and $l$.

Thus log-concavity of $P(t)$ should be approximated by the log-concavity of

$$
Y_{l}(t)=Z_{l}(t) \cdot Z_{l}(1 / t) .
$$

We make this precise. Let now $C_{n}=\left[Y_{l}\right]_{l-n}$ for $0 \leq n \leq l$. We have

$$
C_{n}=\frac{|z|^{l+2+n}-|z|^{l-n}}{|z|^{2}-1} .
$$


We now regard $P_{k, l}$ as a perturbation of $Z_{l}$. Precisely, two coefficients of $Z_{l}$ are perturbed, by (10), by a factor $1+\varepsilon$, resp. $1+\varepsilon^{\prime}$, where $\varepsilon, \varepsilon^{\prime}>0$ are independent of $l$ (Keep in mind that $k$ has been fixed when $l$ can be taken arbitrarily large). Observe that then, since $Z_{l}$ are positive polynomials, when regarding $P$ as a perturbation of $Y_{l}$, the coefficients of $Y_{l}$ are perturbed by a factor at most $1+\varepsilon^{\prime \prime}$, where

$$
\varepsilon^{\prime \prime} \text { is independent of } l \text {, and } \varepsilon^{\prime \prime} \rightarrow 0 \text { for } \varepsilon, \varepsilon^{\prime} \rightarrow 0 \text {. }
$$

Let $l>n>0$. A direct calculation from (11) shows

$$
C_{n}^{2}-C_{n+1} C_{n-1}=|z|^{2 l} \geq \delta \cdot C_{n},
$$

where $\delta>0$ is a constant depending on $z$ but not on $n$ or $l$. Moreover,

$$
\frac{C_{n \pm 1}}{C_{n}}=|z|^{ \pm 1} \cdot \frac{1-|z|^{-2 n-2 \mp 2}}{1-|z|^{-2 n-2}},
$$

which are quantities bounded away from 0 and $\infty$ in a way independent of $n$ and $l$.

Thus if $\varepsilon, \varepsilon^{\prime}, \varepsilon^{\prime \prime}>0$ are small and independent of $l$, then from (13) we have

$$
(1+\varepsilon)^{2} C_{n}^{2}-\left(1+\varepsilon^{\prime}\right) C_{n+1}\left(1+\varepsilon^{\prime \prime}\right) C_{n-1}>0
$$

independently of $n$ and $l$. And we achieved such perturbation of $Z_{l}$ in (12) (where $\varepsilon, \varepsilon^{\prime}, \varepsilon^{\prime \prime}$ have a slightly different meaning).

By using (14) for $n=l-1$, we can find an $l$ sufficiently large, so that $C_{l}>$ $(1+\varepsilon) C_{l-1}$ for any $0<\varepsilon<|z|-1$ fixed before $l$. Then, during the perturbation of $Y_{l}$ to $P$, there is no difficulty either to ascertain that $[P]_{0}>[P]_{1}$, which is the final condition needed to have $P$ being strictly log-concave.

Remark 2. For trapezoidal (rather than log-concave) polynomials the argument can be further drastically simplified. In (5) we can argue with $t+1 / t$ instead of $t^{k}$ and $\frac{t^{l+1 / 2}-t^{-1 / 2-l}}{t^{1 / 2}-t^{-1 / 2}}$ instead of $Z_{l}(t)$, and then the crunching with the various perturbations in Lemma 1 can be skipped.

Remark 3 . Note that by Lemma 2, Theorem 1 readily extends to links of any (fixed) number of components.

Remark 4. By modifying (9) into

$$
Q_{q}=\left(\langle q \cdot P\rangle+t^{l+1}+t^{-1-l}\right) \cdot t^{-1} \cdot(1+t)^{2}+1,
$$

we can achieve the approximation result also for monic polynomials, i.e., such with leading (and trailing) coefficient \pm 1 . Then, by [16], we may choose the knots mentioned in Remark 1 to be additionally fibered.

Acknowledgement. I wish to thank to K. Murasugi for some motivating discussion. 


\section{References}

[1] G. Burde, Das Alexanderpolynom der Knoten mit zwei Brücken, Arch. Math. (Basel) 44 (1985), no. 2, 180-189.

[2] R. Crowell, Genus of alternating link types, Ann. of Math. 69 (1959), no. 2, 258-275.

[3] S. Garoufalidis, Does the Jones polynomial determine the signature of a knot?, preprint, math.GT/0310203.

[4] R. I. Hartley, On two-bridged knot polynomials, J. Austral. Math. Soc. Ser. A 28 (1979), no. 2, 241-249.

[5] X. Jin, F. Zhang, F. Dong, and E. G. Tay, Zeros of the Jones polynomial are dense in the complex plane, Electron. J. Combin. 17 (2010), no. 1, Research Paper 94, 10 pp.

[6] I. D. Jong, Alexander polynomials of alternating knots of genus two, Osaka J. Math. 46 (2009), no. 2, 353-371.

[7] — Alexander polynomials of alternating knots of genus two II, J. Knot Theory Ramifications 19 (2010), no. 8, 1075-1092.

[8] L. Lyubich and K. Murasugi, On zeros of the Alexander polynomial of an alternating knot, Topology Appl. 159 (2012), no. 1, 290-303.

[9] W. W. Menasco, Closed incompressible surfaces in alternating knot and link complements, Topology 23 (1984), no. 1, 37-44.

[10] K. Murasugi, On the genus of the alternating knot. I. II., J. Math. Soc. Japan 10 (1958), 94-105, 235-248.

[11] On the Alexander polynomial of alternating algebraic knots, J. Austral. Math. Soc. Ser. A 39 (1985), no. 3, 317-333.

[12] P. S. Ozsváth and Z. Szabó, Holomorphic disks and knot invariants, Adv. Math. 186 (2004), no. 1, 58-116.

[13] A. Stoimenow, Diagram genus, generators and applications, preprint, arXiv:1101.3390.

[14] , Newton-like polynomials of links, Enseign. Math. (2) 51 (2005), no. 3-4, 211230.

[15] Knots of (canonical) genus two, Fund. Math. 200 (2008), no. 1, 1-67.

[16] Realizing Alexander polynomials by hyperbolic links, Expos. Math. 28 (2010), no. 2, 133-178.

Gwanguu Institute of Science and Technology School of General Studies GwanguU 500-712, Korea

E-mail address: stoimeno@stoimenov.net 